\title{
Increased Cerebral Blood Flow with Increased Amyloid burden in the preclinical phase of Alzheimer's disease
}

\author{
Amir Fazlollahi, PhD, ${ }^{1}$ Fernando Calamante, $\mathrm{PhD},{ }^{2,3,7}$ Xiaoyun Liang,PhD, ${ }^{3,4}$ Pierrick \\ Bourgeat, PhD, ${ }^{1}$ Parnesh Raniga, PhD, ${ }^{1}$ Vincent Dore, PhD, ${ }^{1,6}$ Jurgen Fripp ,PhD, ${ }^{1}$ David \\ Ames, MD, ${ }^{5}$ Colin L. Masters, MD, ${ }^{5,7}$, Christopher C. Rowe, MD, ${ }^{5,6}$ Alan Connelly, \\ $\mathrm{PhD},{ }^{3,7}$ Victor L. Villemagne, MD, ${ }^{3,5}$, Olivier Salvado, $\mathrm{PhD},{ }^{1,8}$ for the Australian Imaging \\ Biomarkers and Lifestyle (AIBL) Research Group. \\ (1) CSIRO Health and Biosecurity, Brisbane, Australia \\ (2) The University of Sydney, Sydney Imaging and School of Aerospace, Mechanical and \\ Mechatronic Engineering, Sydney, Australia \\ (3) Florey Institute of Neuroscience and Mental Health, Heidelberg, Australia
}

(4) Mary MacKillop Institute for Health Research, Australian Catholic University, Melbourne, Australia

(5) The University of Melbourne, Parkville, Australia

(6) Austin Health, Heidelberg, Australia

(7) Florey Department of Neuroscience \& Mental Health, The University of Melbourne, Australia

(8) CSIRO Data61, Sydney, Australia

Corresponding Author: Amir Fazlollahi, Level 5 UQ Health Science Building 901/16, Royal Brisbane and Women's Hospital, Herston QLD 4029,

Email: fazlollahi@gmail.com

Phone: +61405292473 , +6173253 3618

This is the author manuscript accepted for publication and has undergone full peer review but has not been through the copyediting, typesetting, pagination and proofreading process, which may lead to differences between this version and the Version of Record. Please cite this article as doi: $10.1002 / \mathrm{jmri} .26810$

This article is protected by copyright. All rights reserved. 


\section{Acknowledgement}

We are grateful to Drs. Maria Fernandez-Seara (University of Navarra, Spain) and David Feinberg (Advanced MRI Technologies, USA) for providing the initial 3D GRASE pCASL sequence. We acknowledge the facilities of the National Imaging Facility, a National Collaborative Research Infrastructure Strategy (NCRIS) capability, at The Florey Institute of Neuroscience and Mental Health.

Running Title: Perfusion increases with amyloid in pre-AD

\section{Increased Cerebral Blood Flow with Increased Amyloid burden in the preclinical phase of Alzheimer's disease}

Background: Arterial spin labeling (ASL) is an emerging MRI technique for non-invasive measurement of cerebral blood flow (CBF) that has been used to show hemodynamic 
changes in the brains of people with Alzheimer's disease (AD). CBF changes have been measured using PET across the AD spectrum, but ASL showed limited success in measuring $\mathrm{CBF}$ variations in the preclinical phase of $\mathrm{AD}$, where Amyloid $\beta(\mathrm{A} \beta)$ plaques accumulate in the decades prior to symptom onset.

Purpose: The aim of this study is to investigate the relationship between CBF measured by multiphase-pseudo-continuous-ASL (MP-PCASL) and A $\beta$ burden as measured by ${ }^{11} \mathrm{C}-\mathrm{PiB}$ PET imaging in a study of cognitively normal (CN) subjects aged over 65 .

Study type: Cross-sectional

Population: Forty-six cognitively normal subjects including 33 with low levels of $A \beta$ burden and 13 with high levels of $A \beta$.

Field Strength/Sequence: 3 T/ 3D multiphase-pseudo-continuous-ASL (MP-PCASL)

Assessment: The MP-PCASL method was chosen because it has a high signal-to-noise ratio. Furthermore, the data were analyzed using an efficient processing pipeline consisting of motion correction, ASL motion correction imprecision removal, temporal and spatial filtering, and partial volume effect correction.

Statistical Tests: General Linear model

Results: In $C N$ subjects positive for $A \beta$ burden $(n=13)$ we observed a positive correlation between $\mathrm{CBF}$ and $\mathrm{A} \beta$ burden in the hippocampus, amygdala, caudate $(p<0.01)$, frontal, temporal and insula $(p<0.05)$.

Data Conclusion: To the best of our knowledge, this is the first study using MP-PCASL in the study of $\mathrm{AD}$, and the results suggest a potential compensatory hemodynamic mechanism that protects against pathology in the early stages of AD.

This article is protected by copyright. All rights reserved. 


\section{Keywords:}

Perfusion, Multiphase PCASL, preclinical Alzheimer's disease, CBF, amyloid 
ALZHEIMER'S DISEASE (AD) IS the most common cause of dementia ( 70\%) and is characterized by Amyloid $\beta$ (A $\beta$ ) depositions and tau tangles pathologies. ${ }^{1}$ So far, treatments targeting $\mathrm{A} \beta$ protein have had very limited results for $\mathrm{AD}$ patients, ${ }^{2,3}$ and interest has shifted to the pre-clinical phase (asymptomatic phase with the presence of amyloid pathology as defined in ${ }^{4}$ ). The pre-clinical phase may provide an opportunity for effective interventions. In the pre-clinical phase of $\mathrm{AD}$, regional cerebral metabolism and blood flow are altered: metabolism as measured by glucose ${ }^{5-8}$ and cerebral blood flow (CBF) when measured by positron emission tomography (PET) ${ }^{9}$ or arterial spin labelling (ASL) MRI. ${ }^{10}$

Indeed, cerebral blood flow (CBF) as an indicator of brain dysfunction is a well-established biomarker of $\mathrm{AD} .{ }^{11} \mathrm{CBF}$ varies across the $\mathrm{AD}$ spectrum from cognitively normal (CN), to mild cognitive impairment (MCI), to the clinical diagnosis of AD. Based on gold standard PET imaging, there are regions with increased ${ }^{6,9}$ and decreased ${ }^{8}$ activity in CN, increased ${ }^{12}$ and decreased ${ }^{13,14}$ activity in patients with MCI, and decreased ${ }^{15,16}$ regional activity in patients with AD.

Studies investigating brain function changes in the preclinical phase of AD in association with $A \beta$ are limited. In the preclinical phase of AD using PET imaging, a few studies have investigated the mutual effect of CBF and $\mathrm{A} \beta$ deposition. Sojkova et al. investigated whether $\mathrm{A} \beta$ status (+/-) is related to longitudinal CBF changes measured by ${ }^{15} \mathrm{O}-\mathrm{H}_{2} \mathrm{O}-\mathrm{PET}$ in $28 \mathrm{CN}$ subjects, and found hyper-perfusion in the medial frontal, inferior frontal gyri, precuneus, inferior parietal lobule and postcentral gyrus and hypo-perfusion in anterior and middle 


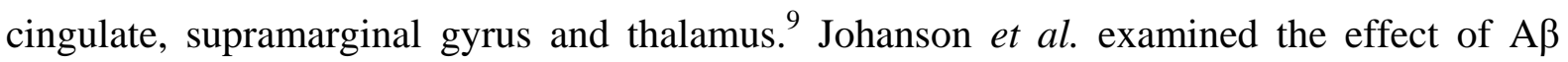
burden and metabolism in a subset of $200 \mathrm{CN}$ subjects, and found that relative to the $\mathrm{A} \beta$ group, $A \beta+$ and $A \beta i$ (intermediate) participants had increased glucose metabolism measured by ${ }^{18} \mathrm{~F}$-FDG-PET in the bilateral thalamus and bilateral superior temporal gyrus. ${ }^{6}$ Other recent studies found a positive correlation between cortical $A \beta$ and metabolism measured by ${ }^{18}$ F-FDG-PET in CN in temporal, parietal and frontal regions, ${ }^{5,7}$ whereas another study showed a significant negative trend only in the medial temporal lobe. ${ }^{8}$

Due to the cost and radiation risk associated with PET imaging, ASL-MRI technique is becoming a popular alternative for measuring CBF. ASL is less expensive, non-invasive, relatively fast (less than 10 minutes) and, with the improvement in MRI hardware and software technology, can be performed on most MRI scanners with comparable results to ${ }^{15} \mathrm{O}-\mathrm{H}_{2} \mathrm{O}$ for $\mathrm{CBF}$ quantification. ${ }^{17,18}$ However, there is a large variability in terms of sensitivity across the many ASL variants, namely pulsed-ASL (PASL), continuous-ASL (CASL), pseudo-continuous-ASL (PCASL), and multiphase-pseudo-continuous-ASL (MPPCASL). ${ }^{19}$ Among these, PCASL and its variants were reported to outperform other methods (higher SNR and less labeling artifacts), ${ }^{20,21}$ and are currently the recommended ASL method by the ISMRM perfusion community and the European consortium for ASL in dementia. ${ }^{22}$

In the preclinical phase of $\mathrm{AD}$ using ASL imaging, there is limited and contradicting evidence of CBF increase. Mattsson et al. reported that a higher cortical A $\beta$ load measured by ${ }^{18}$ F-florbetapir PET imaging was associated with reduced CBF measured by PASL across 
diagnostic groups (CN, MCI and AD) but found no significant difference between $\mathrm{CN} A \beta+$ and $\mathrm{CN} A \beta-$ groups. ${ }^{23}$ In another study using ${ }^{11} \mathrm{C}-\mathrm{PiB}$ PET and PCASL imaging techniques, a lower $\mathrm{CBF}$ was associated with higher $\mathrm{A} \beta$ load in $\mathrm{CN}$ and amnestic MCI. ${ }^{10}$

ASL techniques broadly suffer from low SNR and measurement instabilities, ${ }^{24}$ which may contribute to the inconsistent findings across the dementia research. In this study, by taking advantage of MP-PCASL technique with GRASE readout and background suppression, higher SNR and lower spatial distortion could be achieved. ${ }^{20,25}$ Additional processing with a well-established ASL post-processing workflow also improves the precision of CBF measurements. $^{26}$

The aim of this study was to investigate the associations of CBF measured by MP-PCASL MRI and $\mathrm{A} \beta$ burden as measured by ${ }^{11} \mathrm{C}$-PiB PET imaging in a cross-sectional design with the focus on CN participants.

\section{Material and methods}

\section{Participants}

The data used for this study were obtained from the Australian Imaging, Biomarkers and Lifestyle (AIBL) study. ${ }^{27}$ Written informed consent was obtained from participants in accordance with the Austin Health human research ethics committee. MCI participants met 
the criteria for subjective and objective cognitive difficulties in the absence of manifest functional loss. AD participants met National Institute of Neurological and Communicative Disorders and Stroke-Alzheimer's Disease and Related Disorders Association (NINCDSADRDA) criteria for probable $\mathrm{AD} .{ }^{28} \mathrm{CN}$ older adults were recruited from advertisements and their cognitive health was confirmed by their performance within normal limits on the AIBL neuropsychological test battery and unremarkable medical history. ${ }^{27}$

The cross-sectional data from $46 \mathrm{CN}$ participants. The characteristics of the study population are summarized in Table $\mathbf{1}$.

\section{MRI Acquisition}

All MR images were acquired on a 3T Siemens Tim Trio scanner with a 12-channel head coil. All subject underwent anatomical T1-weighted (T1w) imaging and MP-PCASL with background suppression and 3D GRASE readout. ${ }^{29}$ The T1w images were acquired using a standard 3D magnetization-prepared rapid gradient echo (MPRAGE) sequence with $1 \times 1 \times 1$ $\mathrm{mm}^{3}$ resolution, TR/TE/TI $=1900 / 2.55 / 900 \mathrm{~ms}$, flip angle $9^{\circ}$, field of view $256 \times 256$, and 160 slices.

For the MP-PCASL labeling, 40 pairs of conventional PCASL (with RF offset phases of $0^{\circ}$ for tag $\left(\mathrm{T}_{0}\right)$ and $180^{\circ}$ for control $\left.\left(\mathrm{C}_{0}\right)\right)$ were collected. Subsequently, 20 ASL pairs with $+90^{\circ}$ shift in phase offset $\left(\mathrm{T}_{90}\right.$ and $\left.\mathrm{C}_{90}\right)$ relative to conventional PCASL were also acquired. For all datasets, the labeling plane was selected using an MR angiogram. For data acquisition, a 3D

GRASE PCASL sequence with k-space sharing to improve brain coverage was used. ${ }^{29}$ The 
relevant imaging parameters were: TR/TE of 3750/56 ms (TR $=4150$ ms was used for both scans from one subject, due to power deposition issues), labeling RF pulse flip angle of $28.5^{\circ}$, RF pulse width $650 \mu$ s, with inter-pulse gap of $450 \mu$ s (spacing between RF pulses of 1070 $\mu \mathrm{s})$, peak/average gradient of $8 / 1 \mathrm{mT} / \mathrm{m}$, peak RF amplitude of $53 \mathrm{mG}$, labeling duration of $1584 \mathrm{~ms}$, post-labeling delay of $1540 \mathrm{~ms}$, and background suppression inversion-times (relative to imaging module) of $1800 \mathrm{~ms}$ and $520 \mathrm{~ms}$. The images have an in-plane resolution of $4 \times 4 \mathrm{~mm}^{2}$ and slice thickness of $6 \mathrm{~mm}$, with a matrix size of $64 \times 51 \times 20$. A proton density (PD) image (often referred to as M0 or calibration image) with 8 repetitions and without labeling and background suppression was also acquired. This image was used for ASL quantification, as well as a reference for multi-modal image registration. The overall acquisition times for MP-PCASL was 10 minutes, and a static tissue suppression level of approximately 90\% was achieved from the background suppression technique.

\section{PET Imaging}

Amyloid PET scans were obtained using the ${ }^{11} \mathrm{C}-\mathrm{PiB}$ ligand on a Philips Allegro scanner. Image acquisition time was 20 minutes, starting 40 minutes after injection of the radiotracer. PET images were processed using an automated region of interest method. ${ }^{30}$ Standardized update values (SUV) for neocortex were calculated within a mask comprised of frontal, superior parietal, lateral temporal, lateral occipital, and anterior and posterior cingulate regions. ${ }^{31}$ A ratio of neocortical SUV to the cerebellar cortex SUV was defined as the SUV 
ratio (SUVR) representing neocortical $\mathrm{A} \beta$ burden, and a value above 1.4 designated amyloid positivity.

\section{Neuropsychological testing}

Several neuropsychological tests were available to study cognitive performance across diagnostic groups. The neuropsychological examination as part of the AIBL study included the Mini-Mental State Examination (MMSE), the California Verbal Learning Test (CVLT), Rey Complex Figure Test with 30-minutes delay (RCFT), Logical Memory II (LMII), Verbal Fluency Animals (VFA) and Letter (VFL). Two composite scores were calculated: a composite episodic memory score by averaging CVLT, LMII and RCFT z-score values, and a composite executive function score by averaging VFL and VFL z-scores.

\section{T1w and ASL image post-processing}

The T1w image was segmented into gray matter (GM), white matter (WM), cerebrospinal fluid (CSF) maps, and brain mask using the expectation maximization segmentation algorithm. T1w data were divided into 73 grey-matter (GM) regions by segmentation propagation using the Neuromorphometrics database (63 subjects, http://www.neuromorphometrics.com/). Subsequently, a rigid alignment between the T1w and M0-ASL calibration image was used to propagate the tissue maps and parcellated regions (a reliable match as 3D GRASE has no image distortion). These small sub-regions were then combined to form cerebral lobes.

This article is protected by copyright. All rights reserved. 
CBF was computed using a previously published in-house processing pipeline, which comprised of motion correction, temporal and spatial denoising, partial volume correction and quantification, as previously detailed in. ${ }^{33}$ Relative CBF (rCBF) maps were computed by intensity normalization using the medial segment of post-central gyrus (with a regression model where the reference region as the independent variable). ${ }^{32}$ From the parcellated GM regions, nine volumetric areas including the neocortex, frontal, parietal, temporal, insula, amygdala, hippocampus, caudate and posterior cingulate, were formed.

For the analysis, subjects with quantitative evaluation index (QEI) less than 0.4 for ASL image were excluded, as they were deemed to have low-quality ASL data. ${ }^{33}$ However, the main results are also reported without any subject exclusion.

\section{Statistical Analysis}

The region of interest analysis was conducted using multiple general linear models to investigate the association of CBF with neocortical SUVR, controlling for age, gender, and APOE $\varepsilon 4$ carrier status. All statistical work was conducted with R (version 3.2.4) and all hypothesis tests were 2-sided.

\section{Results}

This article is protected by copyright. All rights reserved. 
Table 2 presents the association between neuropsychological test and diagnosis. The results show that there are no significant differences in cognitive performance between $\mathrm{CN} A \beta$ - and $\mathrm{CN} \mathrm{A} \beta+$ subjects included in the study. Table 2 also shows no significant difference in terms of hippocampal atrophy between $\mathrm{CN} \mathrm{A} \beta-$ and $\mathrm{CN} \mathrm{A} \beta+$.

Figure 1 shows the overall distribution of CBF and amyloid for four age-matched subjects in CN $\mathrm{A} \beta-$ and $\mathrm{CN} A \beta+$ with rCBF values of 37.3 and 32.8, respectively. Figure 2 compares CBF maps of two CN A $\beta+$ subjects with a different level of neocortical A $\beta$ (1.5 SUVR and 2.46 SUVR where the A $\beta$ cut-off is 1.4) particularly in the hippocampus (overlayed) and anterior insula and frontal regions.

Figure 3 shows the overall association between $\mathrm{rCBF}$ and SUVR. While there were no significant differences in cognitive performance between $\mathrm{CN} \mathrm{A} \beta-$ and $\mathrm{CN} A \beta+$, in the $\mathrm{CN}$ $\mathrm{A} \beta+$ group, a positive correlation between $\mathrm{rCBF}$ and $\mathrm{A} \beta$ burden was observed in the frontal (regression $\beta$ coefficient $=10.5 \pm 4.1, p=0.04)$, temporal $(\beta=11.6 \pm 4.1, p=0.03$ ), and hippocampus $(\beta=14.9 \pm 2.8, \mathrm{p}=0.002)$, amygdala $(\beta=12.8 \pm 3.5, \mathrm{p}=0.008)$, insula $(\beta=11.9 \pm 4.4, p=0.03)$, caudate $(\beta=16.4 \pm 3.0, p=0.001)$ but not in posterior cingulate $(\beta=1.9 \pm 2.8, p=0.158)$.

To study the influence of having considered subject exclusion (when the ASL data quality was deemed to be too low, i.e. QEI $<0.4$ ), Figure 4 shows the corresponding associations between CBF and SUVR without exclusion of any subjects. The excluded subjects included 2 $\mathrm{CN} \mathrm{A} \beta$ - and $1 \mathrm{CN} \mathrm{A} \beta+$. The same qualitative trends as in Figure 3 were observed; the same 
statistically significant associations were found (albeit with lower significance), with the exception of the frontal lobe region, which did not reach significance when low-quality ASL data were included in the analysis.

\section{Discussion}

This study investigated the association of blood flow measured by MP-PCASL-MRI with amyloid- $\beta$ burden assessed using ${ }^{11} \mathrm{C}-\mathrm{PiB}-\mathrm{PET}$ in the preclinical phase of $\mathrm{AD}$, and found a positive correlation in the frontal, temporal, caudate and insular regions. We found that in the early stages of $\mathrm{AD}$, in the absence of brain atrophy or cognitive decline, cerebral perfusion increases with increased $A \beta$ deposition. This increased perfusion may play an important role in the preclinical staging of $\mathrm{AD}$, particularly if those with significant amyloid and hypermetabolism are less likely to be classified as neurodegeneration positive. ${ }^{34}$

To the best of our knowledge, this is the first study using MP-PCASL in the study of Alzheimer's disease. Among available ASL sequences (PASL, CASL and PCASL), PCASL sequence with background suppression and 3D GRASE readout has been nominated as the recommended implementation for the clinical use of ASL. ${ }^{22}$ In our study, we use an ASL variant known as MP-PCASL, which considers 4 phase offsets for tag and control acquisitions, and has been shown to substantially improve the reliability of the perfusion

measurement by allowing to compensate for the labeling efficiency loss in PCASL. ${ }^{26}$ The 
ASL post-processing workflow further improves the reliability of CBF measurements ${ }^{26}$ and we expect that the CBF maps used for this analysis have a higher sensitivity to subtle changes in the pre-clinical phase.

Our study extends previous ASL-CBF studies of AD by reproducing similar results as obtained with PET imaging (as the gold standard method) in the pre-clinical phase of AD. Unlike previous ASL studies, PET-based studies reported patterns of hypermetabolism in an elderly population with amyloid pathology in frontal, ${ }^{5,7}$ parietal and temporal, ${ }^{5,7}$ precuneus and postcentral, ${ }^{9}$ and bilateral thalamus regions. ${ }^{6}$ In the current study, we found a positive correlation between $\mathrm{rCBF}$ and $\mathrm{A} \beta$ burden in the frontal, temporal, hippocampus, amygdala, insula and caudate. Visual comparison of the CBF maps clearly shows the pattern of increased CBF among CN A $\beta+$ subjects with different levels of amyloid burden (Figure 2). Among CN A $\beta$ - older adults, there were no significant relationships between CBF and amyloid, likely due to the narrow dynamic range of amyloid level. Among A $\beta+$ subjects, the positive association was directly linked to amyloid burden as no significant relationship with brain atrophy and cognitive scores could be identified.

Increased CBF might reflect increased brain function. An increase in regional CBF in the preclinical phase of $\mathrm{AD}$ when cognitive performance is preserved (as shown in Table 2), has been suggested as a compensatory response to the accumulation of $\mathrm{A} \beta$ pathology. ${ }^{35}$ Other studies also found significant increased perfusion in non-demented elderly subjects at risk for $\mathrm{AD}$, which was also interpreted as a compensatory mechanism leading to a need for extra 
glucose and oxygen to support neuronal activity. ${ }^{36,37}$ Several functional MRI (fMRI) studies of $\mathrm{CN} \mathrm{A} \beta+$ also reported increased neural activity during cognitive activity in comparison to young people or $\mathrm{CN} \mathrm{A} \beta$ - older participants. ${ }^{38,39,34}$ The identified regions with a significant association between $\mathrm{CBF}$ and $\mathrm{A} \beta$ were also consistent with previous studies reporting the early sign of deterioration in the hippocampus and the posterior cingulate, and with disease progression more extensive in the temporal lobe. ${ }^{40}$ Current results in Figure 3 may suggest that the increased $\mathrm{CBF}$ makes the brain resilient to $\mathrm{A} \beta$ pathology in the preclinical phase of $\mathrm{AD}$, and this increase is lost in the clinical presentation of the disease. However, longitudinal follow up scans to study subjects with low and high $\mathrm{CBF}$ in $\mathrm{CN} A \beta+$ are required. The absence of any associations between $\mathrm{CBF}$ and amyloid in the $\mathrm{CN} A \beta$ - subjects is more challenging to study due to the narrow dynamic range of amyloid values.

The main limitations of the current study are the small cohort size of 46 subjects comprised of $33 \mathrm{CN} \mathrm{A} \beta-$ and $13 \mathrm{CN} \mathrm{A} \beta+$, and the cross-sectional nature of the analysis. Furthermore, the current analysis did not control for diurnal variations, the level of caffeine consumption, or other factors that could affect CBF. However, controlling for these effects should (if they do have an effect at all) decrease the variability of the CBF measurement, and improve the significance of our findings.

Our cross-sectional study revealed an association between $\mathrm{A} \beta$ and $\mathrm{CBF}$ in $\mathrm{CN} A \beta+$ in the absence of any cognitive decline and atrophy (Table 1). At such an early stage, this finding 
suggests that the brain is able to compensate for any stress caused by $A \beta$ accumulation as evidenced by increased CBF. This may represent an ideal time for the administration of therapeutic interventions prior to irreversible neuro-degeneration while the brain is still able to compensate. However, the extent of this CBF compensation is unknown and further studies are needed to elucidate this phenomenon.

In conclusion, this study revealed a positive correlation between cerebral blood flow measured by multiphase PCASL-MRI and $\mathrm{A} \beta$ measured by PET in the hippocampus, amygdala, caudate, frontal, temporal and insula of asymptomatic subjects. This suggests a potential compensatory mechanism against $\mathrm{AD}$ pathology which possible to measure with MRI. However, these cross-sectional findings need to be replicated in a larger cohort and followed longitudinally. 


\section{References}

1. Jack CR, Knopman DS, Jagust WJ, et al. Tracking pathophysiological processes in Alzheimer's disease: An updated hypothetical model of dynamic biomarkers. Lancet Neurol. 2013;12(2):207-216. doi:10.1016/S1474-4422(12)70291-0

2. Slomski A. Clinical Trials Update: $A \beta$-Clearing Drug Fails to Slow Alzheimer Disease. Jama. 2016;315(8):742. doi:10.1001/jama.2016.1081

3. Salloway S, Sperling R, Fox NC, et al. Two Phase 3 Trials of Bapineuzumab in Mildto-Moderate Alzheimer's Disease. N Engl J Med. 2014;370(4):322-333. doi:10.1056/NEJMoa1304839

4. Sperling RA, Aisen PS, Beckett LA, et al. Toward defining the preclinical stages of Alzheimer's disease: Recommendations from the National Institute on AgingAlzheimer's Association workgroups on diagnostic guidelines for Alzheimer's disease. Alzheimer's Dement. 2011;7(3):280-292. doi:10.1016/j.jalz.2011.03.003

5. Oh $\mathrm{H}$, Habeck C, Madison $\mathrm{C}$, Jagust W. Covarying alterations in A $\beta$ deposition, glucose metabolism, and gray matter volume in cognitively normal elderly. Hum Brain Mapp. 2014;35(1):297-308. doi:10.1002/hbm.22173

6. Johnson SC, Christian BT, Okonkwo OC, et al. Amyloid burden and neural function in people at risk for Alzheimer's Disease. Neurobiol Aging. 2014;35(3):576-584. doi:10.1016/j.neurobiolaging.2013.09.028

7. Yi D, Lee DY, Sohn BK, et al. Beta-amyloid associated differential effects of APOE $\varepsilon 4$ on brain metabolism in cognitively normal elderly. Am J Geriatr Psychiatry. 2014;22(10):961-970. doi:10.1016/j.jagp.2013.12.173

8. Knopman DS, Jack CR, Wiste HJ, et al. Selective Worsening of Brain Injury Biomarker Abnormalities in Cognitively Normal Elderly Persons With $\beta$-Amyloidosis. JAMA Neurol. 2013;70(8):1030. doi:10.1001/jamaneurol.2013.182

9. Sojkova J, Beason-Held L, Zhou Y, et al. Longitudinal Cerebral Blood Flow and Amyloid Deposition: An Emerging Pattern? J Nucl Med. 2008;49(9):1465-1471. doi:10.2967/jnumed.108.051946

10. Michels L, Warnock G, Buck A, et al. Arterial spin labeling imaging reveals widespread and $A \beta$-independent reductions in cerebral blood flow in elderly apolipoprotein epsilon-4 carriers. J Cereb Blood Flow Metab. 2016;36(3):581-595. doi:10.1177/0271678X15605847

11. Zlokovic B V. Neurovascular pathways to neurodegeneration in Alzheimer's disease and other disorders. Nat Rev Neurosci. 2011;12(12):723-738. doi:10.1038/nrn3114

12. Beason-Held LL, Goh JO, An Y, et al. Changes in brain function occur years before the onset of cognitive impairment. J Neurosci. 2013;33(46):18008-18014. doi:10.1523/JNEUROSCI.1402-13.2013 
13. Chételat G, Desgranges B, de la Sayette V, Viader F, Eustache F, Baron J-C. Mild cognitive impairment: Can FDG-PET predict who is to rapidly convert to Alzheimer's disease? Neurology. 2003;60(8):1374-1377.

http://www.ncbi.nlm.nih.gov/pubmed/12707450. Accessed November 8, 2018.

14. Nestor PJ, Fryer TD, Smielewski P, Hodges JR. Limbic hypometabolism in Alzheimer's disease and mild cognitive impairment. Ann Neurol. 2003;54(3):343-351. doi:10.1002/ana.10669

15. Bradley KM, O’Sullivan VT, Soper NDW, et al. Cerebral perfusion SPET correlated with Braak pathological stage in Alzheimer's disease. Brain. 2002;125(Pt 8):17721781. http://www.ncbi.nlm.nih.gov/pubmed/12135968. Accessed November 8, 2018.

16. Jagust $\mathrm{W}$, Thisted $\mathrm{R}$, Devous MD, et al. SPECT perfusion imaging in the diagnosis of Alzheimer's disease: a clinical-pathologic study. Neurology. 2001;56(7):950-956. http://www.ncbi.nlm.nih.gov/pubmed/11294935. Accessed November 8, 2018.

17. Bokkers RPH, Bremmer JP, van Berckel BNM, et al. Arterial spin labeling perfusion MRI at multiple delay times: a correlative study with $\mathrm{H}(2)(15) \mathrm{O}$ positron emission tomography in patients with symptomatic carotid artery occlusion. J Cereb Blood Flow Metab. 2010;30(1):222-229. doi:10.1038/jcbfm.2009.204

18. Henriksen OM, Larsson HBW, Hansen AE, Grüner JM, Law I, Rostrup E. Estimation of intersubject variability of cerebral blood flow measurements using MRI and positron emission tomography. J Magn Reson Imaging. 2012;35(6):1290-1299. doi:10.1002/jmri.23579

19. Gevers S, van Osch MJ, Bokkers RPH, et al. Intra- and multicenter reproducibility of pulsed, continuous and pseudo-continuous arterial spin labeling methods for measuring cerebral perfusion. J Cereb Blood Flow Metab. 2011;31(8):1706-1715. doi:10.1038/jcbfm.2011.10

20. Xu G, Rowley HA, Wu G, et al. Reliability and precision of pseudo-continuous arterial spin labeling perfusion MRI on 3.0 T and comparison with 15O-water PET in elderly subjects at risk for Alzheimer's disease. NMR Biomed. 2010;23(3):286-293. doi:10.1002/nbm.1462

21. Zhang K, Herzog H, Mauler J, et al. Comparison of Cerebral Blood Flow Acquired by Simultaneous [ ${ }^{15}$ O]Water Positron Emission Tomography and Arterial Spin Labeling Magnetic Resonance Imaging. J Cereb Blood Flow Metab. 2014;34(8):1373-1380. doi:10.1038/jcbfm.2014.92

22. Alsop DC, Detre JA, Golay X, et al. Recommended implementation of arterial spinlabeled perfusion MRI for clinical applications: A consensus of the ISMRM perfusion study group and the European consortium for ASL in dementia. Magn Reson Med. 2015;73(1):102-116. doi:10.1002/mrm.25197

23. Mattsson N, Tosun D, Insel PS, et al. Association of brain amyloid- $\beta$ with cerebral 
perfusion and structure in Alzheimer's disease and mild cognitive impairment. Brain. 2014;137(5):1550-1561. doi:10.1093/brain/awu043

24. Jahng G-H, Song E, Zhu X-P, Matson GB, Weiner MW, Schuff N. Human Brain: Reliability and Reproducibility of Pulsed Arterial Spin-labeling Perfusion MR Imaging. Radiology. 2005;234(3):909-916. doi:10.1148/radiol.2343031499

25. Sugimori H, Fujima N, Suzuki Y, Hamaguchi H, Sakata M, Kudo K. Evaluation of cerebral blood flow using multi-phase pseudo continuous arterial spin labeling at 3tesla. Magn Reson Imaging. 2015;33(10):1338-1344. doi:10.1016/J.MRI.2015.07.016

26. Fazlollahi A, Bourgeat P, Liang X, et al. Reproducibility of multiphase pseudocontinuous arterial spin labeling and the effect of post-processing analysis methods. NeuroImage. doi:10.1016/j.neuroimage.2015.05.048

27. Ellis KA, Bush AI, Darby D, et al. The Australian Imaging, Biomarkers and Lifestyle (AIBL) study of aging: Methodology and baseline characteristics of 1112 individuals recruited for a longitudinal study of Alzheimer's disease. Int Psychogeriatrics. 2009;21(4):672-687. doi:10.1017/S1041610209009405

28. McKhann G, Drachman D, Folstein M, Katzman R, Price D, Stadlan EM. Clinical diagnosis of Alzheimer's disease: report of the NINCDS-ADRDA Work Group under the auspices of Department of Health and Human Services Task Force on Alzheimer's Disease. Neurology. 1984;34(7):939-944.

http://www.ncbi.nlm.nih.gov/pubmed/6610841. Accessed September 26, 2018.

29. Liang X, Tournier JD, Masterton R, Connelly A, Calamante F. A k-space sharing 3D GRASE pseudocontinuous ASL method for whole-brain resting-state functional connectivity. Int J Imaging Syst Technol. 2012;22(1):37-43. doi:10.1002/ima.22006

30. Dore V, Bourgeat P, Villemagne VL, et al. CapAIBL: Automated reporting of cortical PET quantification without need of MRI on brain surface using a patch-based method. In: Lecture Notes in Computer Science (Including Subseries Lecture Notes in Artificial Intelligence and Lecture Notes in Bioinformatics). Vol 9993 LNCS. Springer International Publishing; 2016:109-116. doi:10.1007/978-3-319-47118-1_14

31. Bourgeat P, Doré V, Fripp J, et al. Implementing the centiloid transformation for 11C$\mathrm{PiB}$ and $\beta$-amyloid 18F-PET tracers using CapAIBL. Neuroimage. 2018;183:387-393. doi:10.1016/J.NEUROIMAGE.2018.08.044

32. Fazlollahi A, Ayton S, Bourgeat P, et al. A Framework to Objectively Identify Reference Regions for Normalizing Quantitative Imaging. In: Medical Image Computing and Computer Assisted Intervention -- MICCAI 2018. Springer; 2018:6572. doi:10.1007/978-3-030-00928-1_8

33. Dolui S, Wolf R, Ali Nabavizadeh S, Wolk D, Detre J. Automated Quality Evaluation Index for 2D ASL CBF Maps. 2017:682.

34. Cohen AD, Klunk WE. Early detection of Alzheimer's disease using PiB and FDG 
PET. Neurobiol Dis. 2014;72(Part A):117-122. doi:10.1016/j.nbd.2014.05.001

35. Dai W, Lopez OL, Carmichael OT, Becker JT, Kuller LH, Gach HM. Mild Cognitive Impairment and Alzheimer Disease: Patterns of Altered Cerebral Blood Flow at MR Imaging. Radiology. 2009;250(3):856-866. doi:10.1148/radiol.2503080751

36. Zlatar ZZ, Wierenga CE, Bangen KJ, Liu TT, Jak AJ. Increased Hippocampal Blood Flow in Sedentary Older Adults at Genetic Risk for Alzheimer's Disease. J Alzheimer's Dis. 2014;41(3):809-817. doi:10.3233/JAD-132252

37. Bangen KJ, Restom K, Liu TT, et al. Assessment of Alzheimer's Disease Risk with Functional Magnetic Resonance Imaging: An Arterial Spin Labeling Study. Mandal PK, ed. J Alzheimer's Dis. 2012;31(s3):S59-S74. doi:10.3233/JAD-2012-120292

38. Sperling RA, LaViolette PS, O’Keefe K, et al. Amyloid Deposition Is Associated with Impaired Default Network Function in Older Persons without Dementia. Neuron. 2009;63(2):178-188. doi:10.1016/j.neuron.2009.07.003

39. Mormino EC, Brandel MG, Madison CM, Marks S, Baker SL, Jagust WJ.

A Deposition in Aging Is Associated with Increases in Brain Activation during Successful Memory Encoding. Cereb Cortex. 2012;22(8):1813-1823. doi:10.1093/cercor/bhr255

40. Doré V, Villemagne VL, Bourgeat P, et al. Cross-sectional and Longitudinal Analysis of the Relationship Between A $\beta$ Deposition, Cortical Thickness, and Memory in Cognitively Unimpaired Individuals and in Alzheimer Disease. JAMA Neurol. 2013;70(7):903. doi:10.1001/jamaneurol.2013.1062 
Table 1: Subject characteristics

\begin{tabular}{l|cc}
\multicolumn{1}{c}{ CN A ${ }^{-}$} & CN A $\boldsymbol{\beta}^{+}$ \\
\hline \#N & 33 & 13 \\
Age (mean \pm sd) & $78 \pm 7$ & $80 \pm 7$ \\
Sex (female/male) & $19 / 14$ & $6 / 7$ \\
MMSE (mean \pm sd) & $29.2 \pm 1$ & $28.6 \pm 1$ \\
CDR & $0 \pm 0$ & $0 \pm 0$ \\
APOE $\varepsilon 4(+/-)$ & $4 / 29$ & $5 / 8$ \\
NeoCortical SUVR & $1.27 \pm 0.1$ & $1.88 \pm 0.3^{*}$ \\
NecCortical rCBF & $21.5 \pm 5.8$ & $19.16 \pm 5.1$ \\
$* p<0.05$ & \\
CN = Cognitively Normal; A $\beta=$ Amyloid $\beta$; APOE $\varepsilon 4$ \\
positive = presence of at least one $\varepsilon 4$ allele; SUVR $=$ \\
standardized uptake value ratio; Neocortical CBF is the \\
relative CBF adjusted for age, gender and ApoE status
\end{tabular}

This article is protected by copyright. All rights reserved. 
Table 2: Association of Cognitive Neuropsychological scores and hippocampal atrophy with subject demographics \& diagnosis.

\begin{tabular}{l|cccccccc}
\hline \multicolumn{1}{c}{} & \multicolumn{1}{c}{ Episodic Memory } & \multicolumn{2}{c}{ Executive Function } & Composite Score & \multicolumn{2}{c}{ Hippocampal Atrophy } \\
\hline & \multicolumn{1}{c}{$\boldsymbol{\beta}$} & $\boldsymbol{P}$ & $\boldsymbol{\beta}$ & $\boldsymbol{P}$ & $\boldsymbol{\beta}$ & $\boldsymbol{P}$ & $\boldsymbol{\beta}$ & $\boldsymbol{P}$ \\
Age & -0.029 & 0.16 & 0.004 & 0.85 & 0.004 & 0.85 & $\mathbf{- 4 4 . 1}$ & $\mathbf{0 . 0 0 3}$ \\
Sex & -0.175 & 0.55 & 0.418 & 0.16 & 0.205 & 0.46 & 110.6 & 0.571 \\
APOE: $\varepsilon 4$ & -0.152 & 0.70 & 0.99 & 0.80 & 0.103 & 0.79 & -366.9 & 0.176 \\
Diagnosis: & -0.296 & 0.4 & 0.30 & 0.93 & -0.202 & 0.57 & 338.6 & 0.167 \\
CN+ & & & & & & & &
\end{tabular}

$\beta$ : regression beta coefficients, $P$ : $p$-value 


\title{
Increased Cerebral Blood Flow with Increased Amyloid burden in the preclinical phase of Alzheimer's disease
}

\author{
Amir Fazlollahi, $\mathrm{PhD},{ }^{1}$ Fernando Calamante, $\mathrm{PhD},{ }^{2,3,7}$ Xiaoyun Liang,PhD, ${ }^{3,4}$ Pierrick \\ Bourgeat, PhD, ${ }^{1}$ Parnesh Raniga, PhD, ${ }^{1}$ Vincent Dore, PhD, ${ }^{1,6}$ Jurgen Fripp ,PhD, ${ }^{1}$ David \\ Ames, MD, ${ }^{5}$ Colin L. Masters, MD, 5,7, Christopher C. Rowe, MD, ${ }^{5,6}$ Alan Connelly, \\ $\mathrm{PhD},{ }^{3,7}$ Victor L. Villemagne, MD, ${ }^{3,5}$, Olivier Salvado, $\mathrm{PhD},{ }^{1,8}$ for the Australian Imaging \\ Biomarkers and Lifestyle (AIBL) Research Group. \\ (1) CSIRO Health and Biosecurity, Brisbane, Australia \\ (2) The University of Sydney, Sydney Imaging and School of Aerospace, Mechanical and \\ Mechatronic Engineering, Sydney, Australia \\ (3) Florey Institute of Neuroscience and Mental Health, Heidelberg, Australia
}

(4) Mary MacKillop Institute for Health Research, Australian Catholic University, Melbourne, Australia

(5) The University of Melbourne, Parkville, Australia

(6) Austin Health, Heidelberg, Australia

(7) Florey Department of Neuroscience \& Mental Health, The University of Melbourne, Australia

(8) CSIRO Data61, Sydney, Australia

Corresponding Author: Amir Fazlollahi, Level 5 UQ Health Science Building 901/16, Royal Brisbane and Women's Hospital, Herston QLD 4029, Email: fazlollahi@gmail.com

Phone: +61405292473 , +6173253 3618

\section{Acknowledgement}

This article is protected by copyright. All rights reserved. 
We are grateful to Drs. Maria Fernandez-Seara (University of Navarra, Spain) and David Feinberg (Advanced MRI Technologies, USA) for providing the initial 3D GRASE pCASL sequence. We acknowledge the facilities of the National Imaging Facility, a National Collaborative Research Infrastructure Strategy (NCRIS) capability, at The Florey Institute of Neuroscience and Mental Health.

Running Title: Perfusion increases with amyloid in pre-AD 


\section{CN A $\beta-\quad$ CN A $\beta+$}
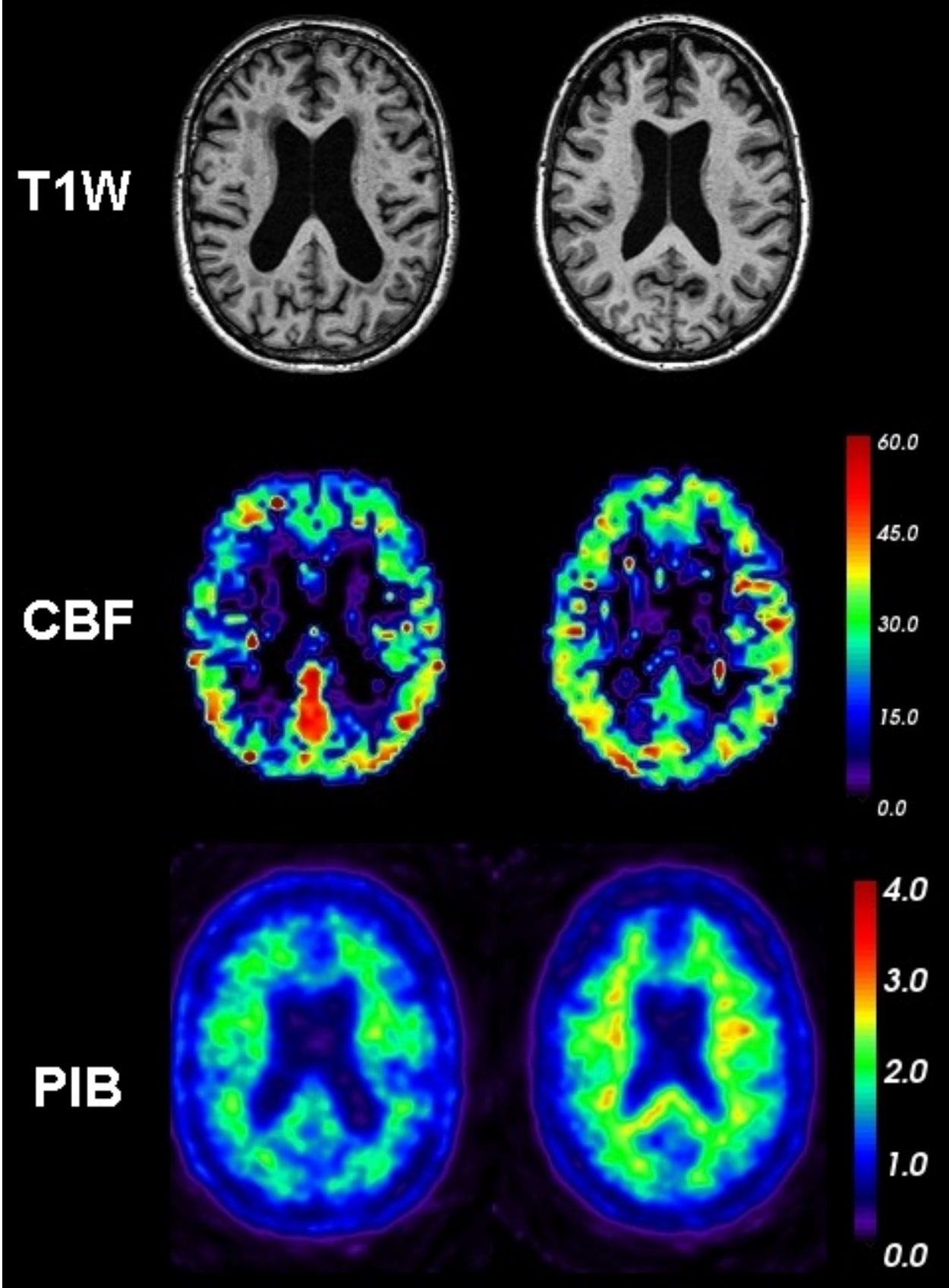

JMRI_26810_Fig1_CBF_PIB_T1W.tif 


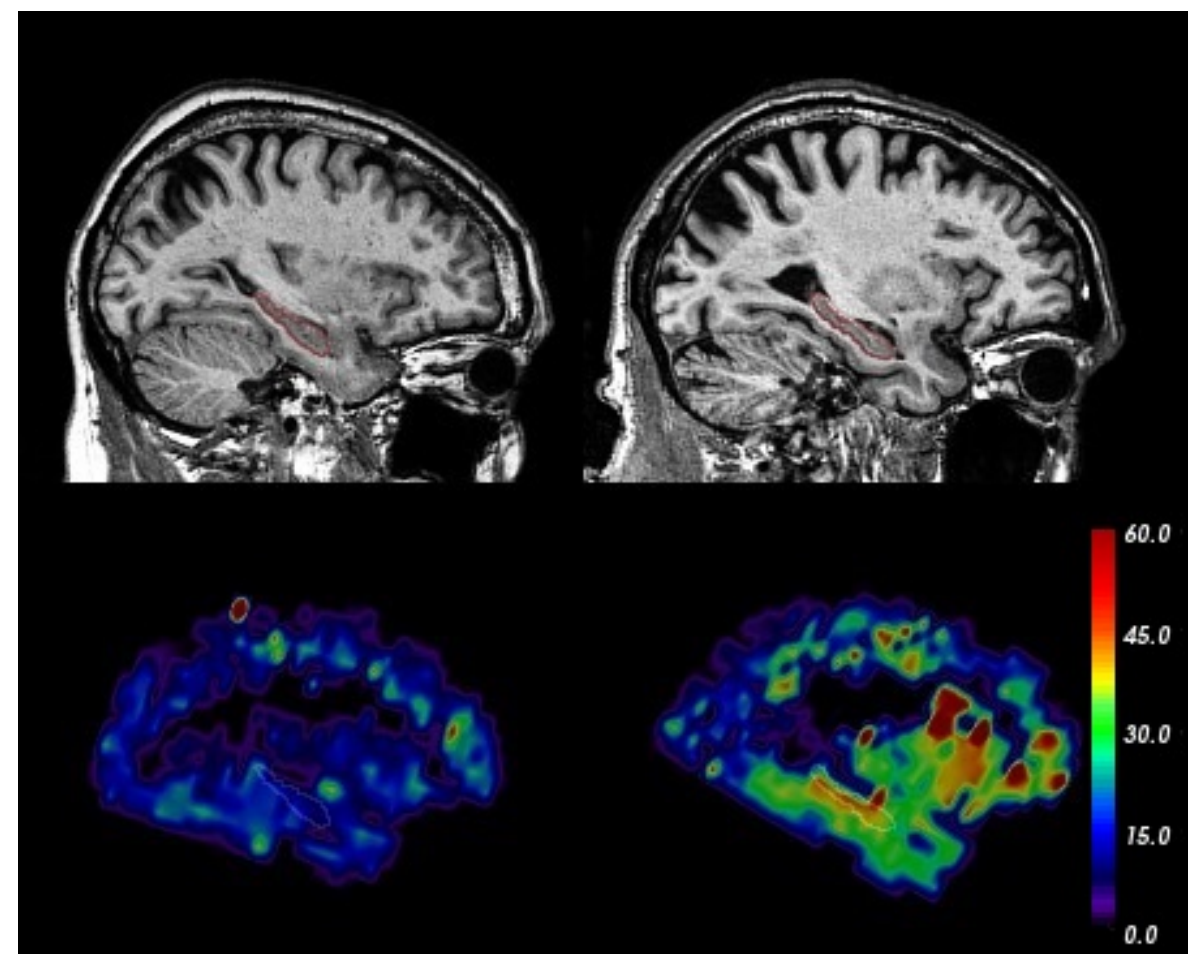

JMRI_26810_Fig2_CBF_T1W_Hippo.tif

This article is protected by copyright. All rights reserved. 
Neocortex

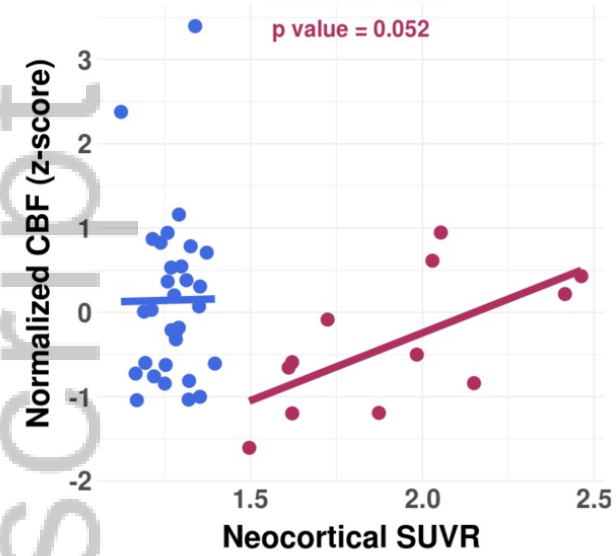

Temporal Lobe

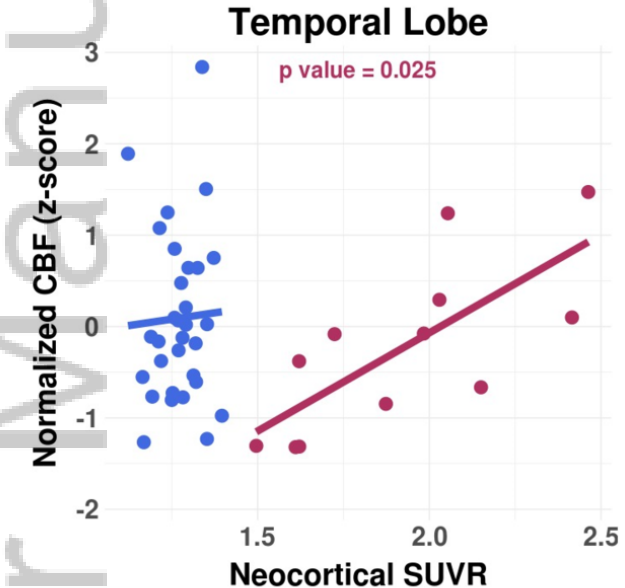

Neocortical SUVR

Hippocampus

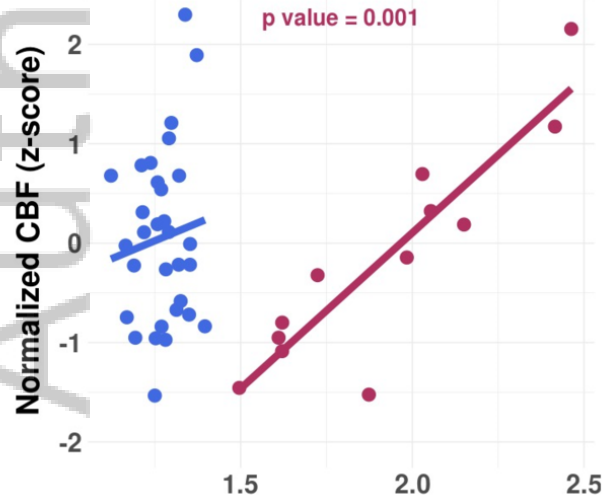

Neocortical SUVR
Frontal Lobe
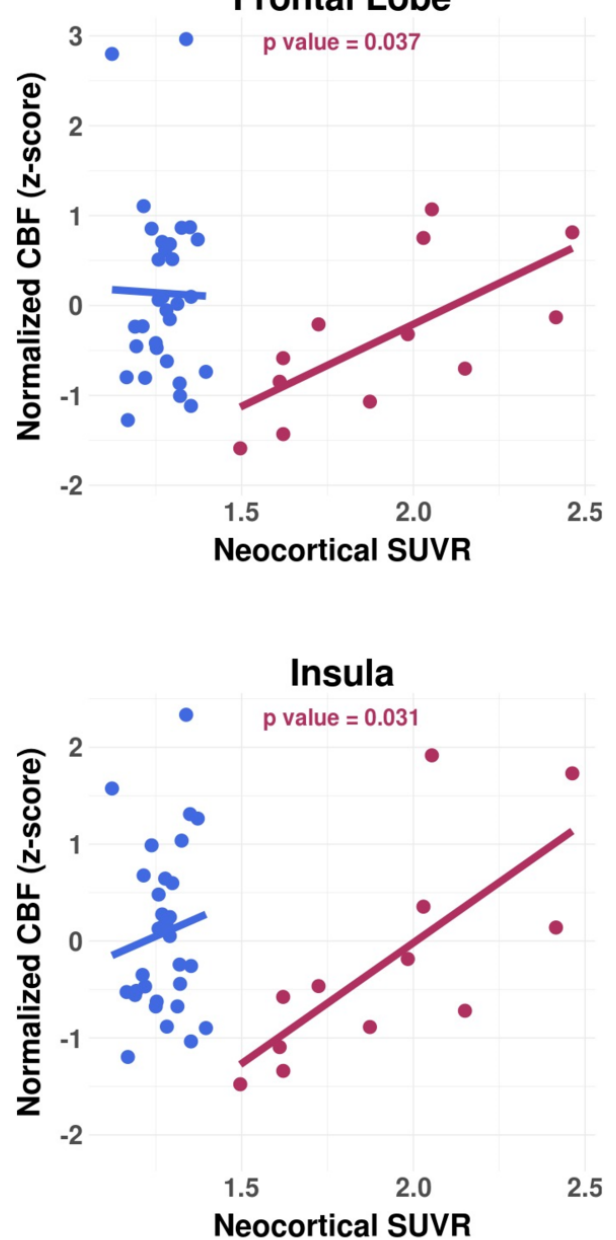

Caudate

$\mathrm{p}$ value $=0.001$

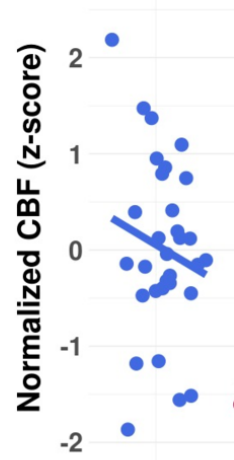

1.5

2.0

Neocortical SUVR
Parietal Lobe

$\mathrm{p}$ value $=0.064$

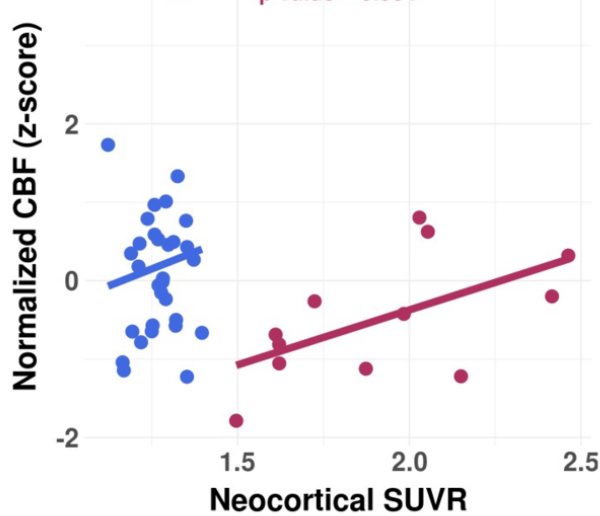

Amygdala

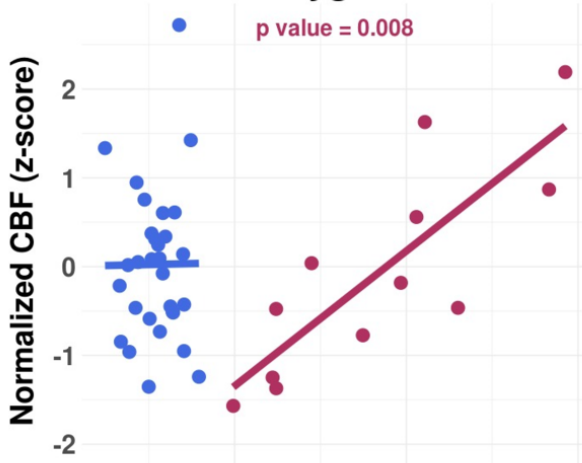

$1.5 \quad 2.0$

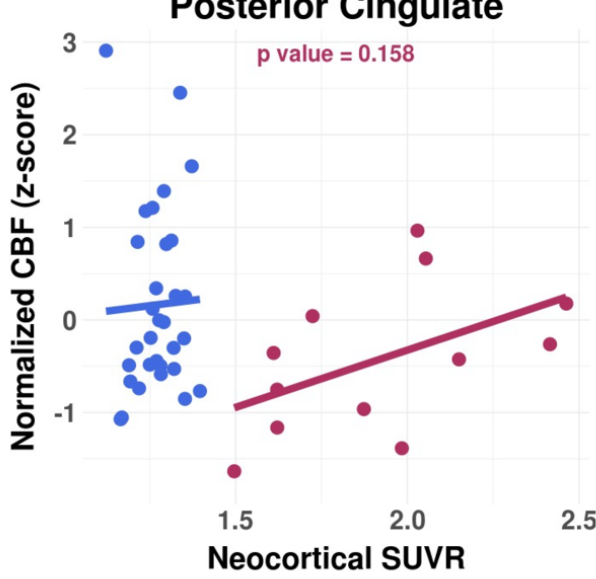

JMRI_26810_mresults_CBF_SUVR.tif 
Neocortex

- $\quad$ v value $=0.248$

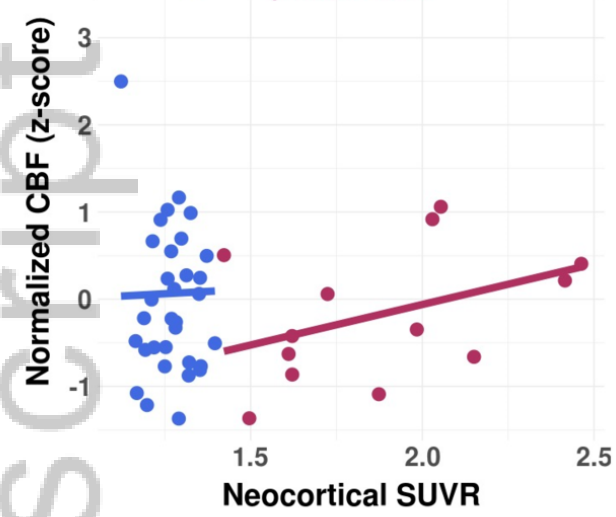

Temporal Lobe

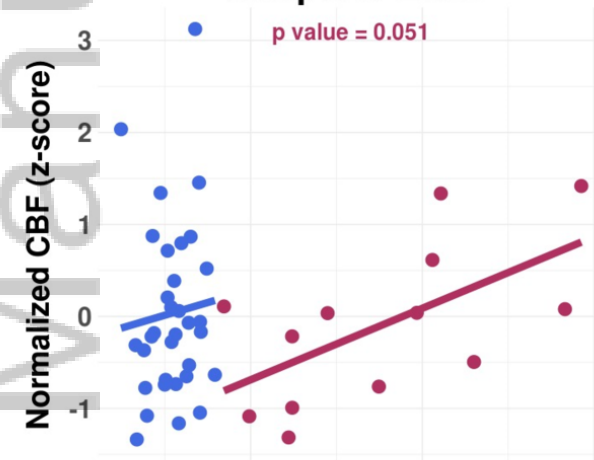

$\begin{array}{ll}1.5 & 2.0 \\ \text { Neocortical SUVR }\end{array}$

Hippocampus

$\mathrm{p}$ value $=0.004$

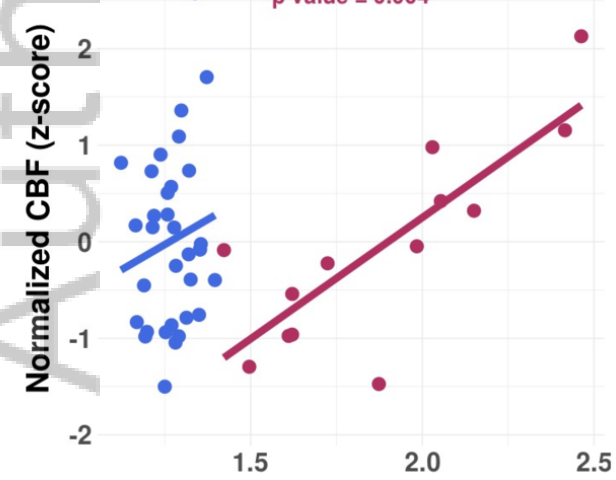

Neocortical SUVR
Frontal Lobe

$p$ value $=0.137$
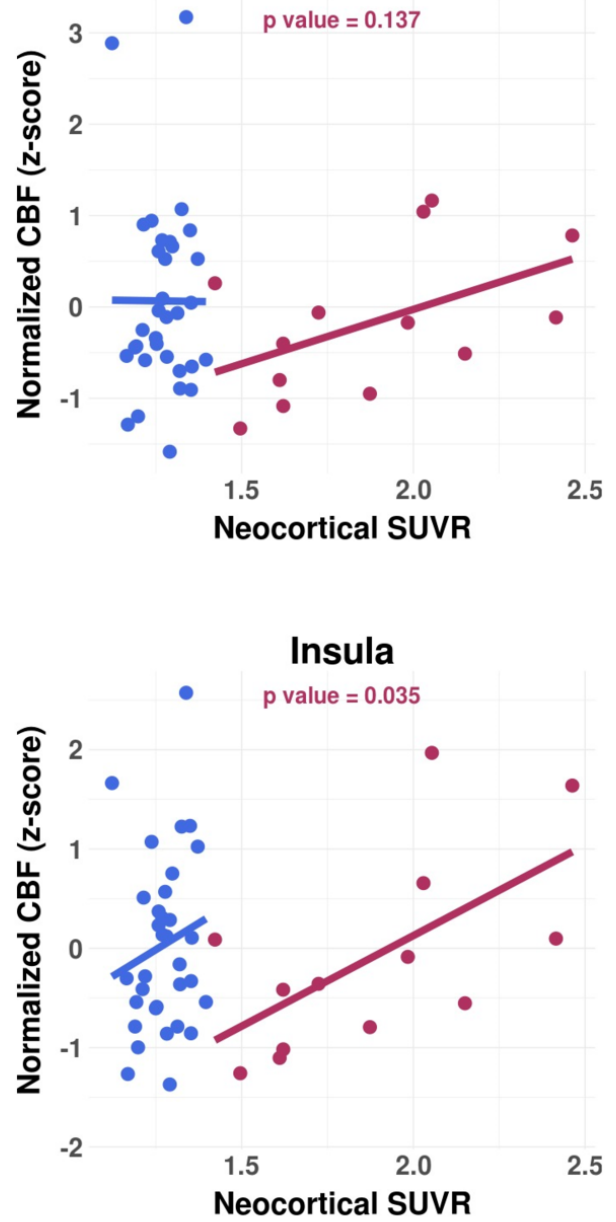

\section{Caudate}

$p$ value $=0.007$

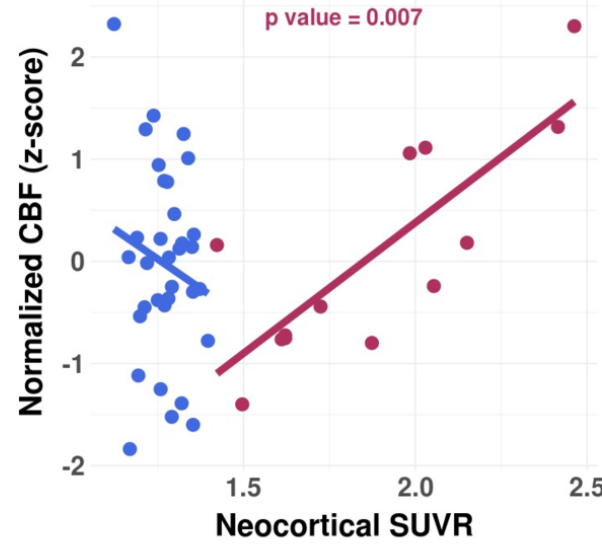

JMRI_26810_mresults_CBF_SUVR_all.tif
Parietal Lobe

$p$ value $=0.305$

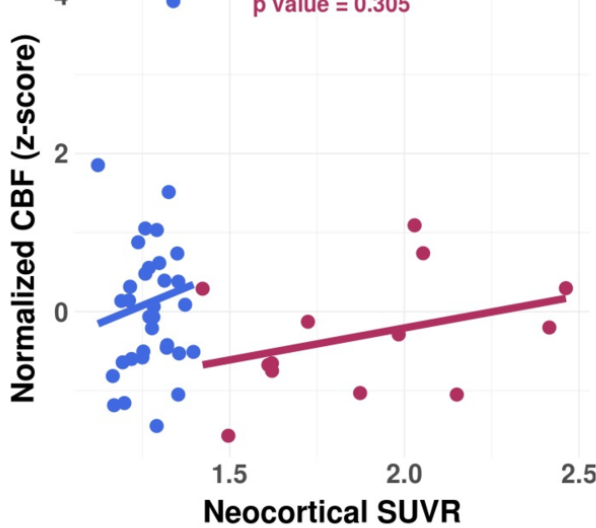

Amygdala

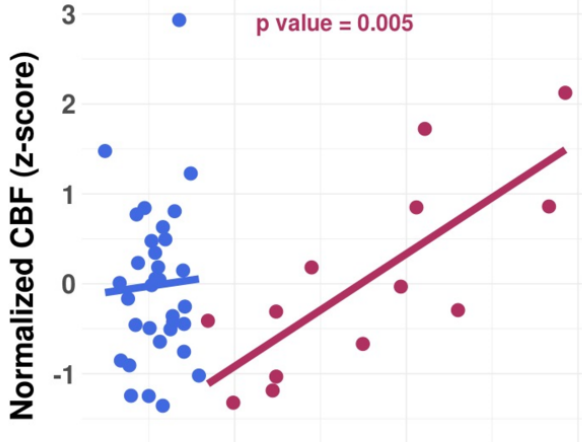

$1.5 \quad 2.0$ Neocortical SUVR

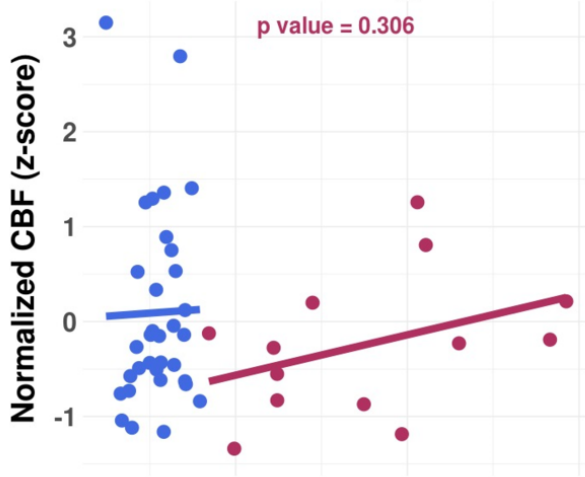

$1.5 \quad 2.0$

Neocortical SUVR
2.5 


\section{University Library}

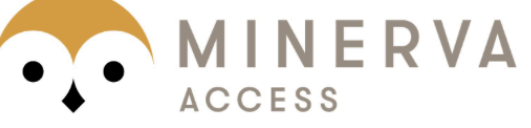

A gateway to Melbourne's research publications

Minerva Access is the Institutional Repository of The University of Melbourne

\section{Author/s:}

Fazlollahi, A;Calamante, F;Liang, X;Bourgeat, P;Raniga, P;Dore, V;Fripp, J;Ames, D;Masters, CL;Rowe, CC;Connelly, A;Villemagne, VL;Salvado, O

Title:

Increased cerebral blood flow with increased amyloid burden in the preclinical phase of alzheimer's disease

Date:

2020-02-01

Citation:

Fazlollahi, A., Calamante, F., Liang, X., Bourgeat, P., Raniga, P., Dore, V., Fripp, J., Ames, D., Masters, C. L., Rowe, C. C., Connelly, A., Villemagne, V. L. \& Salvado, O. (2020). Increased cerebral blood flow with increased amyloid burden in the preclinical phase of alzheimer's disease. JOURNAL OF MAGNETIC RESONANCE IMAGING, 51 (2), pp.505-513. https:// doi.org/10.1002/jmri.26810.

Persistent Link:

http://hdl.handle.net/11343/285926 